\title{
An arbitrary Lagrangian Eulerian (ALE) based numerical method for the computation of gas-particle two phase flow
}

\author{
S. Zhang, M. Zhang, H. Zhou \& J. Xiong \\ Beijing Institute of Applied Physics and Computational Mathematics, \\ China
}

\begin{abstract}
In this paper, an arbitrary Lagrangian Eulerian (ALE) based numerical method has been presented for the numerical simulation of gas-particle two phase flow with moving boundaries. The main stages for the implementation of the algorithm have been discussed. The numerical results of cylindrical implosion and 2D dusty gas explosion have shown the effectiveness of the method.
\end{abstract}

Keywords: ALE method, compatible Lagrangian method, gas-particle two phase flow.

\section{Introduction}

Gas-particle two phase flow is a complex flow phenomenon in the natural environment and industrial engineering. With the development of physical modeling, numerical investigation has been playing a more and more important role in the research field. For most numerical investigation, the physical domain is fixed, thus the Eulerian numerical methods are generally used with fixed grid and fixed boundaries. However, for the investigation of the strong interaction of gas-particle two phase flow with structure where the solid structure deformation is severe and the physical boundary is moving, the numerical method should have the ability to track the moving boundary (also the fluid-structure interface) and a moving grid is need. In this paper, an arbitrary Lagrangian Eulerian (ALE) based numerical method is presented for gas-particle two phase flow with a trajectory model, which satisfies the above requirements $[1,2]$. 
For the investigation of transient dynamic response of complex engineering systems, such as laser and explosion driven problems and fluid-structure interaction problems, the ALE method is the most valuable numerical method. The grid can move with arbitrary speed, which takes advantage of tracking the material interface with the grid. When the grid moves with material speed, the ALE method is just a pure Lagrangian method that is suitable for computational solid mechanics. In this mode, the grid may be distorted under the circumstances of severe shear deformation of the flow field, especially for fluid mechanics; thus, the grid should move at an appropriate speed to keep the grid in good quality to support the computation. A special case is that the grid is fixed without moving, which is just the Eulerian mode of the ALE method.

The object of this paper is the application of the ALE method to gas-particle two phase flow with a two-way coupling trajectory model. After a brief introduction of the ALE method, some key problems are presented for the application of the method in multiphase flow. Two numerical examples have been given to show the effectiveness and robustness of the method.

\section{Numerical method}

In this section, the general ALE method is discussed at first, followed by its application to gas-particle simulation.

The explicit ALE algorithm of this paper can be divided into two stages in general. The first stage is the Lagrangian stage, followed by the rezone and remap stage.

In the first stage the Lagrangian method is the compatible Lagrangian method [3]. The new method is a kind of physically motivated discretization method that can achieve the goal of representing as faithfully and accurately as possible the mathematic and physical property underlying dynamic differential equations while keeping the accuracy and efficiency of the algorithm in a simple and generic manner. The theoretical basis of the method is the support operator method, utilizing the vector identities of differential calculus to derive compatible sets of the fundamental vector differential operators, such as gradient divergence and curl in discrete form. This is first done by first specifying one of these operators (called the prime operator) and then using the vector identities written in discrete summation form to consistently determine the others. Although a finite truncation error is inherent to the method, certain global physical properties, such as the conservation of total energy, can be satisfied to numerical roundoff error, although only the internal equation is to be solved.

The differential equations to be solved can be written in the following form in general for continuous mechanics.

$$
\begin{aligned}
& \frac{1}{V} \frac{d V}{d t}=\nabla \cdot \vec{v} \\
& \rho \frac{d \vec{v}}{d t}=\nabla \cdot \overrightarrow{\vec{\sigma}}
\end{aligned}
$$




$$
\begin{gathered}
\rho \frac{d e}{d t}=\overrightarrow{\vec{\sigma}}: \overrightarrow{\vec{\varepsilon}} \\
\overrightarrow{\vec{\sigma}}=\overrightarrow{\overrightarrow{\vec{C}}}: \nabla \overrightarrow{\vec{v}}
\end{gathered}
$$

where $V(=1 / \rho), \rho, e, \vec{v}, \overrightarrow{\vec{\sigma}}, \overrightarrow{\vec{\varepsilon}}$ are the specific volume, density, internal energy, velocity, stress and strain tensor. Equation (4) is the general constitutive equation.

The compatible Lagrangian method utilizes a staggered grid for space discretization and predictor-corrector two steps for time discretization. For shock problems, artificial viscosity should be used $[4,5]$. The contact and impact algorithm is a better choice if there are complex interactions at the interfaces between different materials.

The second stage is the rezone and remap stage, which is necessary when the grid is in severe distortion [6,7]. The object of the stage is to construct a new grid with much better geometric quality than the old one to conduct the computation smoothly and to project the known flow field in the old grid to the new grid with high accuracy [1].

When the ALE method above is applied to the numerical simulation of gasparticle two phase flow with a two-way coupling trajectory model [8], things are similar to the Eulerian methods for the calculation of the particle phase; the main difference is in the computation of the continuous carrier phase described above. However, two key problems should be addressed with much care [8]. The first one is the searching problem, which requires a fast search technique to determine which cell of the moving unstructured grid a particle with known coordinates locates. The second one is the interaction of the particles with the moving wall of a condensed matter, which may occur in the problem of the interaction of a gasparticle two phase flow with a structure. The particles may bounce from the wall, or slide on the wall, so appropriate models and algorithms are needed.

\section{Numerical method}

Two examples are presented to show the effectiveness of the ALE method of this paper. The first one in fact is a simple example of the interaction of a gas-particle two phase flow with a structure with moving boundaries. The second one is a fixed boundary gas-particle flow, which may be simulated traditionally with the pure Eulerian method, but here is simulated with the ALE method.

\subsection{D cylindrical dusty gas implosion}

Initially, the particle distribution in space is uniform, and the particles with a uniform diameter of $10 \mu \mathrm{m}$ are at rest in the static air at a pressure of $1 \mathrm{~atm}$. The dusty gas is driven by a thin metal shell toward the center with an initial high velocity. The dynamic behavior of the shell is described with an elastic-plastic model. The numerical results are shown in figures 1 to 3 in non-dimensional style. 


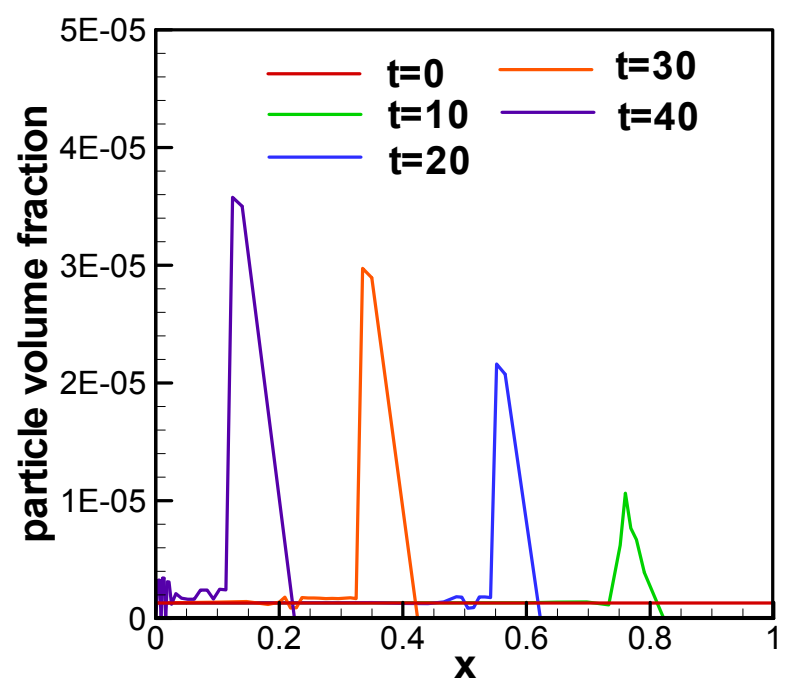

Figure 1: The distribution of the particle volume fractions.

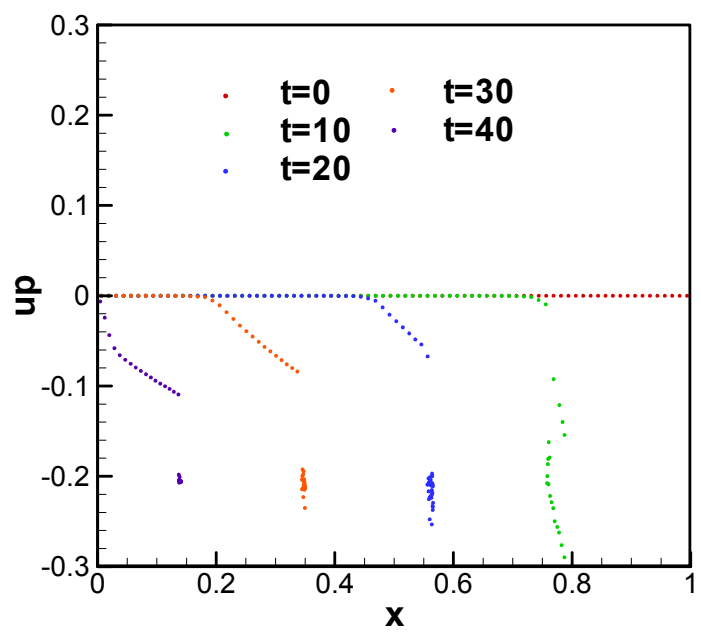

Figure 2: The distribution of the particle velocities. 
Figure 1 shows the distribution of the particle volume fraction in a 1D dusty gas implosion at $t=0,10,20,30$, and 40 . With the moving of the interface, the gas is compressed and the particles move with the gas. The particles near the interface collide with the interface and are accelerated. The particle is accumulated at the region near the surface. The maximal value of the particle volume fraction increases with time during the period of compression. Figure 2 shows the distribution of particle velocity in a $1 \mathrm{D}$ dusty gas implosion at $\mathrm{t}=0$, $10,20,30$, and 40 . The particles near the interface have the maximal velocity. The maximal particle velocity decreases with time. The compressed gas decreases the particle and it results in the increasing of the maximal value of the particle volume fraction with time, as shown in figure 1. Figure 3 shows the distribution of particle temperature in a $1 \mathrm{D}$ dusty gas implosion at $\mathrm{t}=0,10,20$, 30 , and 40 . Due to the compression, the gas near the interface has a higher temperature. Therefore, the temperature of the particles near the interface increases by adsorbing the heat from the surrounding gas.

\subsection{D explosion of dusty gas}

At $t=0$, the ratio of the gas density and pressure is 10 , between the inner and the outer region of the column. The particle concentration is 1 inside the column and 0 outside the column. Two phases are at rest in the beginning. Figure 4 gives the particle concentrations at $\mathrm{t}=0,2$, and 5 . The particle concentration in the center and periphery is high. In addition, the particle concentration is high in the direction perpendicular to the coordinate axis.



Figure 3: The distribution of the particle temperatures. 

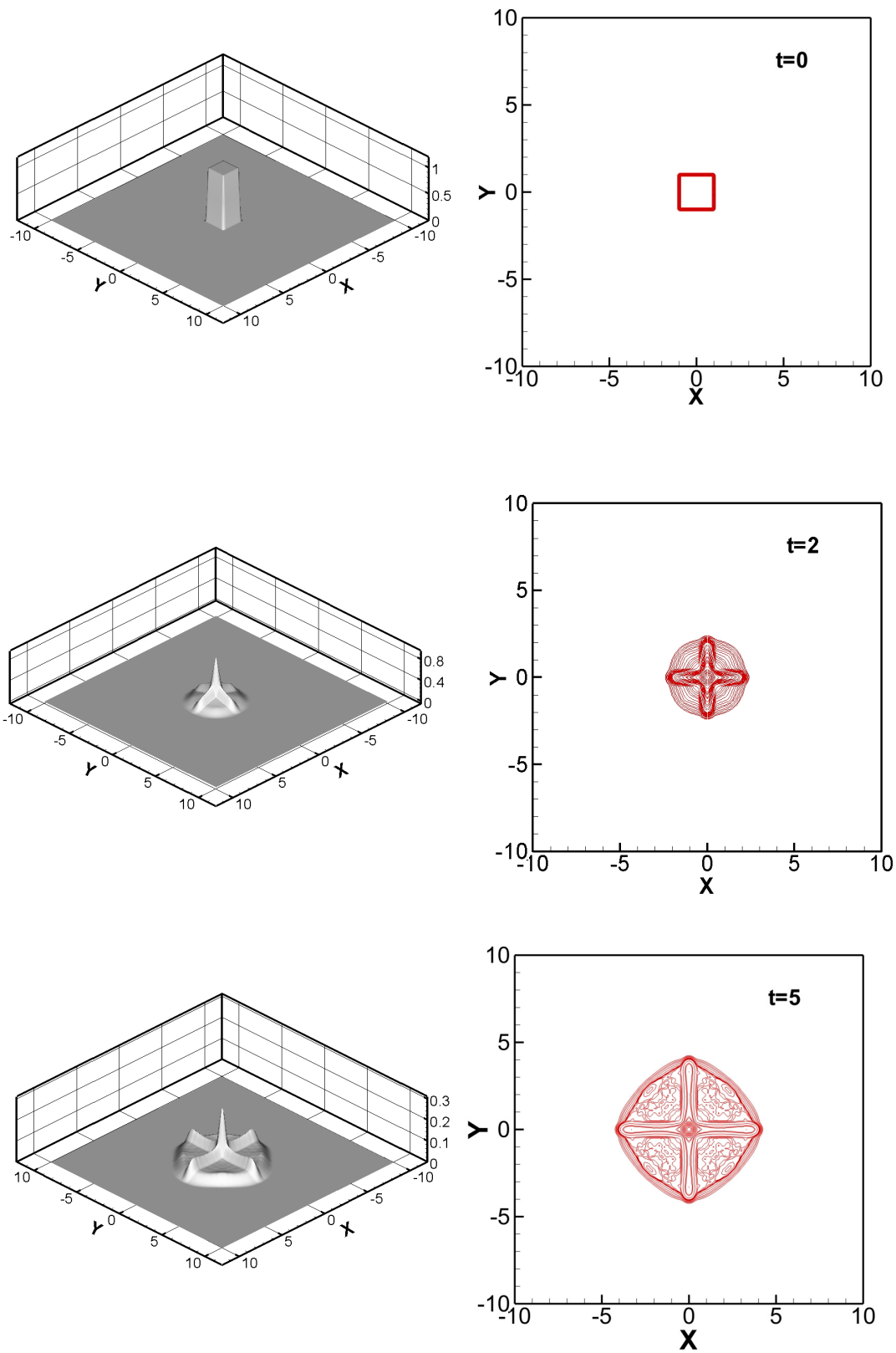

Figure 4: Particle concentration of the $2 \mathrm{D}$ dusty gas explosion: $\mathrm{t}=0.0$ (top); $\mathrm{t}=2.0$ (middle); $\mathrm{t}=5.0$ (bottom). 


\section{Conclusion}

An arbitrary Lagrangian Eulerian (ALE) based numerical method has been presented for the numerical simulation of a gas-particle two phase flow with moving boundaries. This ALE method takes advantage of the recently developed compatible Lagrangian method. A brief introduction was given for the implementation of this algorithm. The numerical results have proved that the new method is effective and robust for the transient analysis of a strong driven gas-particle two phase flow.

\section{References}

[1] Hirt, C.W., Amsden, A.A. and, Cook, J., An arbitrary Lagrangian-Eulerian computing method for all flow speeds. J. Comp. Phys., 14:227-253, 1974. reprinted in 1997, 135: 203-216.

[2] Benson, D.J., Computational methods in Lagrangian and Eulerian hydrycodes. Comput. Methods Appl. Mech. Engrg., 99:235-394, 1992.

[3] Caramana, E.J., Burton, D.E. Shashkov, MJ. and Whalen, P.P., The construction of compatible hydrodynamics algorithms utilizing conservation of total energy. J. Comp. Phys. 146(1):227-262, 1998.

[4] Caramana, E.J., Shashkov, M. J. and Whalen, P.P., Formulations of artificial viscosity for multi-dimensional shock wave computations. J. Comp. Phys., 144(1):70-97, 1998.

[5] Haibing Zhou, Jun Xiong and Shudao Zhang., Formulation of Artificial Viscosity for Lagrangian Analysis of Shocks. The 8th World Congress on Computational Mechanics (WCCM8) and the 5th European Congress on Computational Methods in Applied Sciences and Engineering (ECCOMAS 2008), June 30 -July 5, 2008, Venice, Italy.

[6] Canann, S.A., Tristano, J.R., Staten, M.L., An approach to combined Laplacian and optimization-based smoothing for triangular, quadrilateral, and quad-dominant meshes, in: Proceedings of the Seventh International Meshing Roundtable, Dearborn, MI, pp. 479-494. 1998.

[7] Margolin, L. G. and Shashkov, M. J., Remapping, recovery and repair on a staggered grid. Comput. Methods Appl. Mech. Engrg., 193:4139-4155, 2004.

[8] Mingyu Zhang, Shudao Zhang, Haibin Zhou, Jun Xiong, Numerical Investigation of the Interaction between Shock and the Particles. The paper in this symposium. 